\title{
ON THE USE OF REFLECTIVE WRITING EXERCISES FOR IMPROVING STUDENT LEARNING OF CONCEPTUAL AND TECHNICAL PROBLEMS IN ENGINEERING
}

\author{
Lawrence R. Chen, Maxime Jacques, and Zeinab Sobhanigavgani \\ Department of Electrical and Computer Engineering, McGill University, Montreal, QC Canada \\ lawrence.chen@mcgill.ca, maxime.jacques@mail.mcgill.ca, zeinab.sobhanigavgani@mail.mcgill.ca
}

\begin{abstract}
Self-reflection and reflective writing are often used to promote self-regulated learning amongst students (Nilson, 2013). A number of engineering programs are incorporating greater opportunities for student reflection (Turns et al., 2014); at the same time, there is a growing need for additional research on the impact of selfreflection and reflective exercises in engineering education (Clark and Dickerson, 2019). We describe the implementation and examine the impact of two types of reflective writing exercises - an exam wrapper and selfevaluation-in two Electrical and Computer Engineering courses, a fundamental first year course on signals and systems and a final year technical elective course on photonics.
\end{abstract}

Keywords: Self-reflection, reflective exercises, selfregulated learning, instructional strategies.

\section{INTRODUCTION}

Self-regulated learning, which involves adapting one's learning activities strategically and intentionally to achieve specific learning objectives, has been shown to improve student performance and expand depth of thinking [1-3]. Self-reflection and reflective writing ${ }^{1}$ are often used to encourage self-regulated and life-long learning skills.

Recently, it has been recognized that engineering programs should include more opportunities for student reflection $[4,5]$. A number of studies on the impact of selfreflection in project-based and design courses, e.g., capstone design courses, with regards to students' design abilities have been conducted [6,7]. For example, Adams et al. found that senior engineering students were more characteristic of 'reflective practitioners', i.e., practising reflection-in-action (in the design process), and considered a greater breadth of information and issues when addressing complex design tasks [6]. Self-reflection has also been used in non-technical engineering courses to

\footnotetext{
${ }^{1}$ We refer to reflective writing and reflective writing exercises as written summaries of reflections.
}

provide students with an opportunity to develop a greater appreciation for professional practice and values, as well as the social responsibilities of engineers [8]. However, there are fewer research publications that examine the impact of reflection on student learning of conceptual and technical ideas in courses that are not focused on design and/or the design process $[9,10]$. In one study, Benson and Zhu use self-assessment (SA) in two core mechanical engineering courses, a first-year Introduction to Engineering course and an advanced Solid Mechanics course, to allow students to determine weaknesses and develop strategies to improve their performance on tests [9]. While they found that students in the advanced course provided more elaborate reflections, "it is unclear whether the reflection exercise helps students repair their gaps or simply prompts the student to pause and reflect on the possible cause of their error...” [9]. In a second study, Clark and Dickerson explore the impact of reflection-onaction (after an activity) to enhance students' post-activity understanding in electrical and computer engineering courses on Digital and Analog Electronics [10]. They found that the courses using reflective exercises were associated with better student performance on final exams, though the difference compared to the courses not employing reflection was not large.

In this paper, we examine the impact of two types of reflective writing exercises in two electrical and computer engineering courses: a fundamental first year course on signals and systems and a final year technical elective course on photonics. Our study follows those conducted in [9] and [10] that focus on the use of reflection to enhance student learning of conceptual and technical issues.

\section{COURSE DETAILS}

Reflective writing exercises were used in two undergraduate courses in electrical and computer engineering: ECSE 206 Introduction to Signals and 
Systems and ECSE 430 Photonic Devices and Systems / ECSE 540 Photonic Devices and Applications.

\subsection{ECSE 206}

ECSE 206 is a fundamental core course taken by electrical and computer engineering students. The course provides an introduction to continuous time (CT) and discrete time (DT) signals and systems, their representations in the time, spectral (e.g., Fourier series and Fourier transforms), and transform domains (e.g., Laplace and $\mathrm{z}$ ), and the connection between CT and DT via sampling. Specific topics include:

- Review of complex functions;

- Discrete and continuous time signals, basic system properties;

- Linear time-invariant systems, convolution;

- Fourier series and Fourier transforms, frequencydomain analysis, filtering, sampling;

- Laplace transforms and inversion, transfer functions, poles and zeros, solutions of linear constantcoefficient differential equations, transient and steady-state response;

- Z-transforms.

Students will have previously taken a conventional mathematics course on ordinary different equations as well as an introductory course on electric circuits.

In the Fall 2018 semester, the enrolment was 52 students. The course was taught using a flipped classroom approach: content was delivered via short videos and class time (3 hours per week, run by the instructor) was spent reviewing key concepts, testing students through quizzes and in-class polling questions, providing opportunities for peer instruction, and having students practice through problem solving. Problem solving was reinforced through additional practice during tutorials (2 hours per week, run by a teaching assistant (TA)). Assessments (\% of final grade) included reflective writing exercises (3\%), pre-/ post-class online quizzes (5\%), in-class quizzes (7\%), submission of class activities involving individual and/or group work (27\%), a video problem (3\%), one midterm test (25\%), and a two-part final (30\%) involving both individual and group work.

\subsection{ECSE 430 / ECSE 540}

ECSE 430 is a final year technical elective course taken by electrical engineering students. During the Fall 2018 semester, ECSE 430 was co-taught with ECSE 540, a graduate version of the course (broadly speaking, both courses have the same learning outcomes). The course introduces photonic devices and their applications and specific topics include:

- Semiconductor lasers, optical amplifiers, optical modulators;

- Photodetectors and optical receivers;
- Optical fibers and waveguides, fiber and waveguide devices;

- Photonic systems (communications, sensing, biomedical);

- Optical test-and-measurement instrumentation with experiments on characterizing photonic devices and systems.

In terms of background, undergraduate students will have completed courses on electromagnetism and electromagnetic waves; they will also have seen some basic concepts of waves and physical optics in their freshmanlevel physics courses. On the other hand, the background of graduate students is highly variable; for some students, this is the first course that they took in photonics while others had familiarity with some or most of the topics.

The total enrolment was 30 students -17 undergraduates and 13 graduates. The course was also taught using a flipped classroom approach. Students were expected to learn material on their own ahead of class through assigned readings in textbooks and/or course notes; class time was devoted to reviewing concepts, inclass polling questions, peer instruction, problem solving activities, and occasional content delivery. Formally, the class involved 3 hours of lecture and 2 hours of tutorial per week; however, there was no distinction between the lectures and tutorial. The 5 hours of contact time were run by the instructor with occasional assistance from the TA. Assessments (\% of final grade) included reflective writing exercises (5\%), pre-/post-class online quizzes (5\%), 5 assignments (30\%), and 4 in-class tests (60\%).

\section{REFLECTIVE WRITING EXERCISES AND EVALUATION}

Reflective writing exercises were used to provide students with an opportunity to engage in self-direction and self-evaluation, as well as a means to promote a better understanding of specific conceptual and technical topics. Both courses implemented two types of reflective writing exercises: exam wrappers (EWs) and SAs.

\subsection{Exam Wrapper}

EWs are reflective exercises used to engage students in reflection following a test. In our implementation, the graded tests were returned to the students in the class immediately following, e.g., within 24 or 72 hours. During class, a quick overview of the test was provided and then students were asked to complete a questionnaire to guide their reflection. The questions were designed to allow students to reflect on gaps in their knowledge of concepts and technical content. Specifically, students were asked to complete or respond to the following:

1. Go through the different questions and highlight directly on the test your error(s); explain your error(s) and/or any misunderstanding/misconception that led 
to the incorrect response(s). Do you now understand the source of your misunderstanding/misconception?

2. How did you prepare for this test?

3. How confident were you about your knowledge of the material before taking the test? And after taking the test?

4. Which question in the test was most challenging for you? Why do you think that is?

5. Based on your response to the question above (i.e., \#4), does this question resemble a question previously covered, e.g., in a class activity or on an assignment? What is different between this test question and the previous question(s)?

6. What changes do you need to make to prepare for the next test? Is this realistic?

In ECSE 206, students completed one EW after the midterm while in ECSE 430 / ECSE 540, students completed four EWs, one after each in-class test.

\subsection{Self-Assessment}

In this exercise, students responded to a series of questions that were again designed to guide their reflection. In ECSE 206, while the specific points to address or questions to answer for each reflective exercise varied depending on the topic being covered at the time, they revolved around three principle themes:

1. To summarize important concepts and/or identify similarities between topics.

2. To describe how well they understand concepts and what issues they find confusing.

3. To explain how they are consolidating knowledge and filling any gaps.

In ECSE 430 / ECSE 540, the specific points to address and questions to answer were the same for all reflective writing exercises. In particular, students were asked to describe the following:

1. How well they felt they achieved the learning objectives or outcomes associated with a specific section of the course and/or how well they understood a specific topic.

2. Their confidence level with being able to solve various types of problems (analysis, design) associated with the topic.

3. In what areas they felt they needed additional work, what they needed to do to improve their understanding, and how they would go about trying to consolidate their knowledge.

In ECSE 206, there were 5 SAs that were assigned throughout the semester. In ECSE 430 / ECSE 540, there were 4 SAs that were assigned after the in-class tests; they were intended to supplement the reflections from the EWs and focused on specific technical issues that might not have related to questions on the tests.

\subsection{Implementation Details}

In both courses, the EWs comprised a written questionnaire that students completed and uploaded via a learning management system (in our case, myCourses), along with a scanned copy of their marked test. In ECSE 206, completing the EW was worth 2 points out of 40 of the midterm grade while in ECSE 430 / ECSE 540, completing the EW was worth 1 point out of the 30 of the test grade. No specific format was adopted for the SAs; students simply uploaded their responses to the questions on myCourses or via email to the instructor. Students received full credit towards the reflection component of their final grade if they completed $80 \%$ of the SAs, i.e., this was a participative activity and the quality of reflection did not impact the students' grade on the reflective writing exercise.

\section{METHODS}

\subsection{Assessment of Reflection}

The quality of each reflective writing exercise was scored according to a set of rubrics that examined whether the students produced a coherent, relevant, and thoughtful reflection. Specifically, an ideal reflection was defined as one in which the student 'clearly summarizes a thorough and honest effort of self-assessment relative to pre-defined learning objectives, technical concepts, or evaluation questions.' Moreover, the reflection is 'complete, focused and detailed, and identifies clearly problematic matters and specifies a strategy that can be adopted to address weaknesses in understanding or exam preparation.' The length of a student's reflection was not a factor in determining its quality. The reflections were scored on a scale of 0 to 3 , depending on the extent to which the reflection satisfied the following statement, 'the student's reflection aligns well with the description of an ideal reflection':

0 : Strongly disagree

1: Disagree

2: Agree

3: Strongly agree

The rubrics and scoring system were designed to align with the four-category scheme developed by Kember et al. [11]: (1) non-reflection (score of 0), (2) understanding (score of 1), (3) reflection (score of 2), and (4) critical reflection (score of 3). Each reflective writing exercise was scored by the instructor as well as a graduate research assistant who also served as a TA for the course. The scores were then added to obtain a total score out of 6 .

\subsection{ECSE 206}

ECSE 206 is a course that is highly mathematical in nature. While students can acquire skills to perform mechanical computations, they often struggle with a fundamental understanding of concepts and physical 
interpretations (the mathematical concepts remain too abstract). Some examples include:

1. The relationship between causality and stability for linear time invariant systems (continuous or discrete) and the implications on pole-zero location for the transfer function representing the system.

2. The spectral representation of signals, i.e., Fourier series and/or Fourier transforms, as well as transform description of signals, i.e., Laplace or z-transform, and the notion of frequency response (of a system).

The inability to link concrete concepts with mathematical abstractions, e.g., that the Fourier series coefficients of a periodic signal are associated with the harmonics of the signal and can be measured on a spectrum analyzer, hinders the students' ability to solve problems that are not framed as direct mechanical calculations, or that are designed to link concepts together, e.g., how to determine quickly the Fourier transform representation of a periodic signal from its Fourier series representation.

The midterm test and final each contained one part that comprised several true/false questions (with justification) that tested students' comprehension of concepts (denoted Q1M and Q1F, respectively). The midterm test and final also contained one part covering the spectral representations of CT signals (denoted Q3M) and DT signals (denoted Q3F); these questions were not framed as direct mechanical calculations.

One of the SAs (denoted RW1) focused on the students' learning of Fourier series and Fourier transforms for CT signals and to reflect on concepts that were causing confusion / gaps in understanding that they had. This reflection took place before the midterm. A second SA (denoted RW3) focused on the students' ability to identify similarities and differences between the spectral representations of CT and DT signals and what strategies they were using to consolidate their knowledge. We examined the grades obtained on Q3M and Q3F as a function of the quality of RW1 and RW3. Based on the responses in the EW, a number of students identified having difficulty with fundamental concepts (Q1M) and the spectral representation of signals (Q3M). We studied the normalized grade difference between Q1F and Q1M (or Q3F and Q3M) as a function of the quality of the EW. The grades were normalized to the average grade for the question in order to reduce the possible influence of variability in the level of difficulty of the questions between the midterm and final. Finally, for each student, we considered the normalized grade difference between Q1F and Q1M as a function of their average score of the quality for all five SAs.

\subsection{ECSE 430 / ECSE 540}

The methodology for assessing the impact of reflection on student performance in ECSE 430 / ECSE 540 is different from that used in ECSE 206 due to the following: (1) the variability in student background due to the cross- listed nature of the course and (2) the implementation of the reflective writing exercises and in particular, the fact that SAs focused on learning outcomes and were carried out after in-class tests (the in-class tests typically assessed students on the very learning outcomes that they reflected on in the SAs).

For each student, we determined their average score of the quality for all 8 reflective writing exercises (4 EWs and 4 SAs). We then sorted the students into 3 groups based on their average score: (1) Group A (2 graduate students, 5 undergraduate students) with an average score $<3$ (associated with the categories of non-reflection and understanding), (2) Group B (8 graduate students, 7 undergraduate students) with an average score between 3 and 5 (associated with the category of reflection), and (3) Group C (3 graduate students, 5 undergraduate students) with an average score between 5 and 6 (associated with the category of critical reflection).

First, we examined overall course grade as a function of average score of the quality of reflection. Next, for each group of students (i.e., Group A, Group B, and Group C), we examined (1) the average group grade relative to the average class grade for each in-class test and (2) the average group overall course grade relative to the average class overall course grade.

Table 1: Survey questions used in class to gauge student opinion on the reflective writing exercises.

\begin{tabular}{|l|}
\hline Question $1-$ I spend ... minutes doing the reflective \\
writing exercises \\
A. $\quad<1$ min \\
B. $\quad<$ mins \\
C. $\quad<10$ mins \\
D. $\quad>10$ mins \\
\hline Question $2-$ I have referred to my reflective writings... \\
A. Never \\
B. Once \\
C. A few times \\
D. Often \\
\hline Question 3 - Overall, the reflective writing exercises \\
contribute to my learning \\
A. Strongly disagree \\
B. Disagree \\
C. Neither agree nor disagree \\
D. Agree \\
E. Strongly agree \\
\hline Question $4 a(E C S E ~ 206$ only) - I have tried reflective \\
writing in other courses \\
A. Not a chance \\
B. A few times \\
C. A lot \\
\hline Question $4 \boldsymbol{b}$ (ECSE 430 / ECSE 540 only) - I used my \\
reflective writing as a tool when preparing for class or \\
assessments \\
A. Strongly disagree \\
B. Disagree \\
C. Neither agree nor disagree \\
D. Agree \\
E. Strongly agree \\
\hline
\end{tabular}




\subsection{Student Surveys}

The students in both courses were surveyed to gauge their opinion on the reflective writing exercises. The surveys were conducted in-class using TurningPoint and the responses were recorded anonymously; participation was not mandatory. In both courses, approximately 50\% of the students responded to the poll questions, which are shown in Table 1.

\section{RESULTS AND DISCUSSION}

\subsection{ECSE 206}

Generally speaking, as shown in Figure 1, students with a higher quality of reflection on RW1 and RW3 obtained higher grades on questions related to Fourier series and Fourier transforms on the midterm and final. Our observation is that the specific questions relating to the spectral representations of signals were effective at allowing students to learn and understand the material and go beyond simple direct calculation questions.

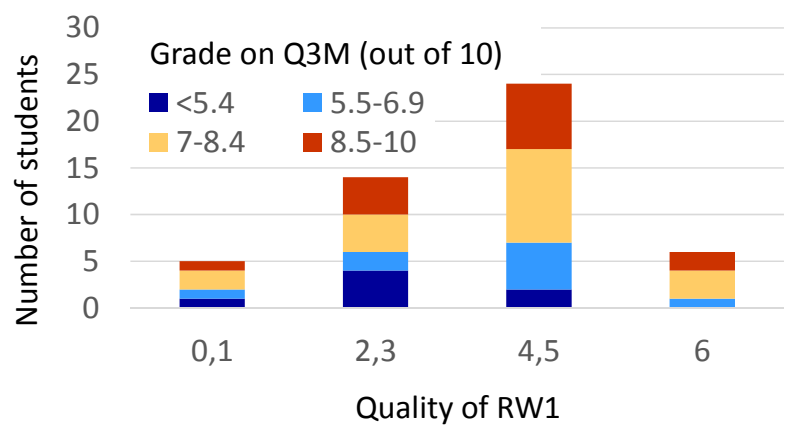

(a)

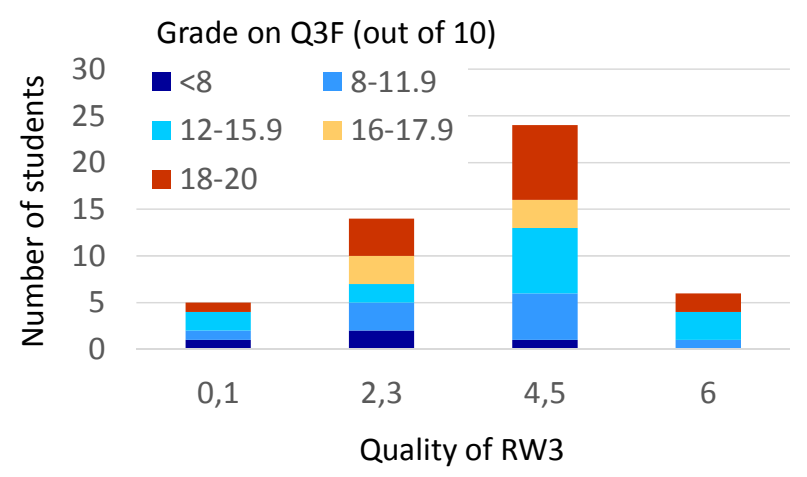

(b)

Fig. 1. Grades on Q3M (a) and Q3F (b) as a function of the quality of RW1 and RW3.

Figure 2 illustrates that students with a higher quality of reflection on the EW also tended to perform better on the final for the type of question that posed them greater difficulty (i.e., the grades on Q1F and Q3F were higher compared to Q1M and Q3M). However, while targeted reflections helped the students identify areas and concepts that they needed to work on, we observe from the results shown in Figure 3 that the reflections over the course of the semester did not translate necessarily into improved performance on conceptual questions, i.e., the grade for Q1F was not generally higher if the average score of the quality for all 5 SAs was higher.

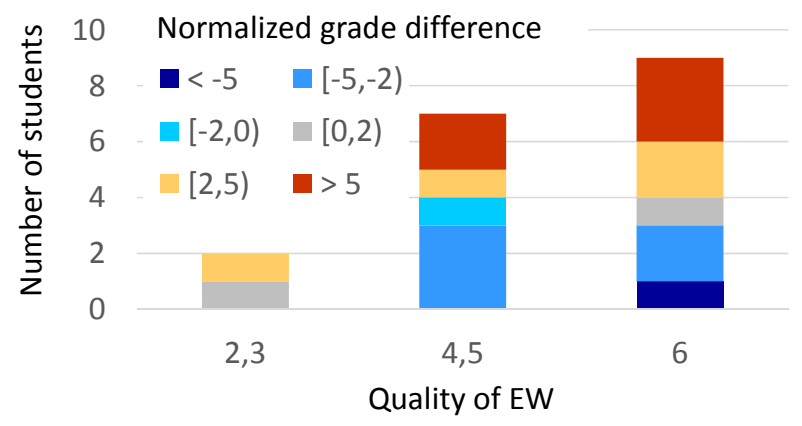

(a)

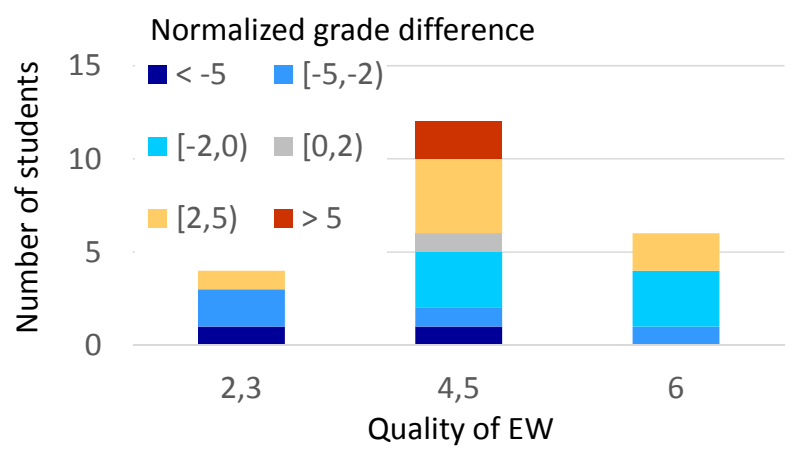

(b)

Fig. 2. Normalized grade difference as a function of quality of the quality of the EW: (a) Q1F / Q1M for students identifying difficulty with Q1F in the EW and (b) Q3F / Q3M for students identifying difficulty with Q3M in the EW.

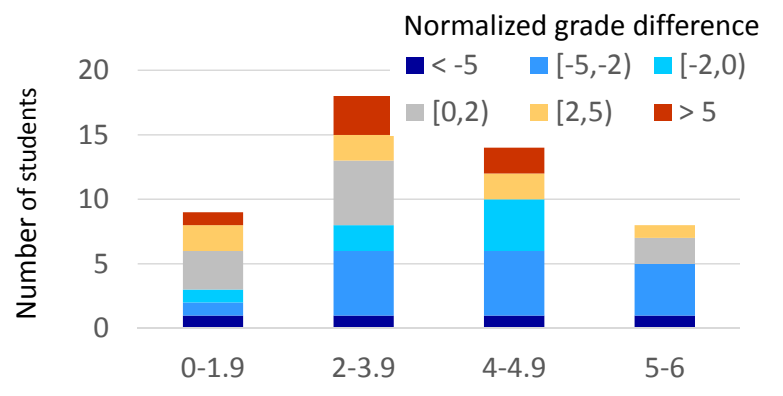

Average score of the quality for all 5 SAs

Fig. 3. Normalized grade difference between Q1F and Q1M as a function of the average score of the quality for all 5 SAs. 


\subsection{ECSE 430 / ECSE 540}

Figure 4 shows that students with a higher average score of the quality for the reflective writing exercises tended to perform better (this is generally expected and the results alone do not prove a causal relation).

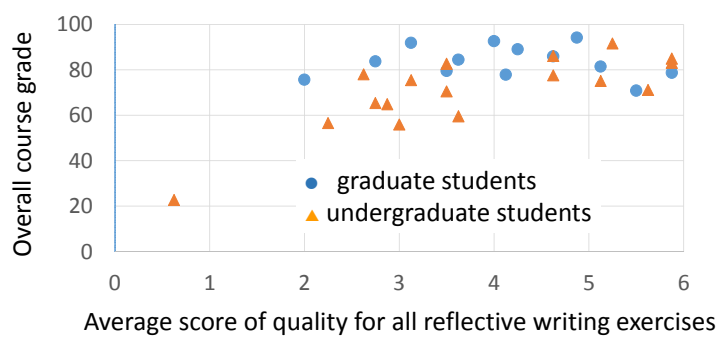

(a)

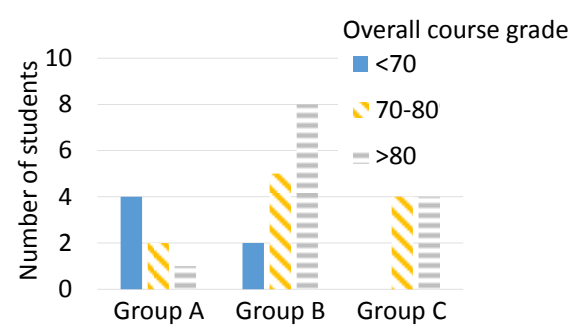

(b)

Fig. 4. (a) Overall course grade as a function of the average score of the quality for all reflective writing exercises and (b) distribution of grades for students in Groups A, B, and C.

Figure 5 shows the difference between the average grades obtained by students in Groups A, B, and C and the average grade of the class for the in-class tests and overall course grade. Students in Group A, especially undergraduate students, performed below the class average on the class tests and had significantly lower overall grades. Undergraduate students in Group B also had grades below the class average on the tests; however, the group average overall course grade was only slightly lower than that of the class. It is clear that students in Group C had the best performance in the in-class tests and overall course grade. We observe that the reflective writing exercises seem to have a more variable impact on undergraduate students compared to graduate students who tended to perform close to or well above the class average. This may be attributed to the broader technical background and experience of the graduate students.

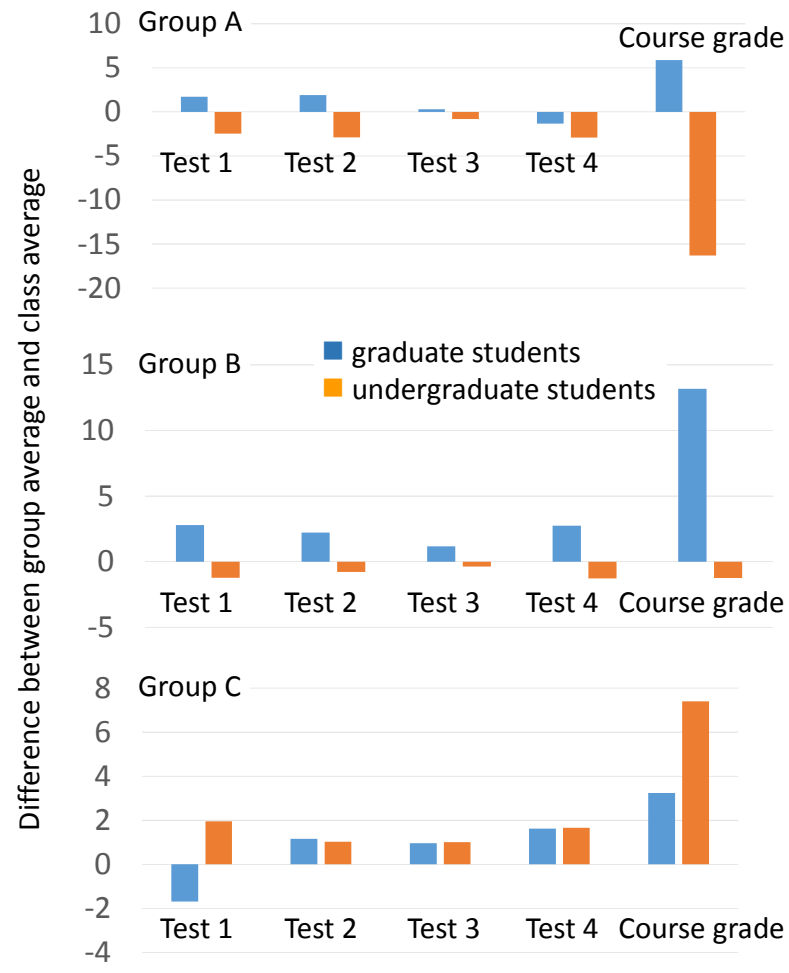

Fig. 5. Difference between group average and class average on in-class tests and overall course grade.

\subsection{Results from Student Surveys}

The results from the student surveys are shown in Figure 6. For both courses, a significant percentage of students take more than 10 minutes to complete each one (Question 1). We infer that the students take the exercise seriously and do not simply write for the purposes of obtaining credit (especially for the SAs). Nearly half of the students found that the reflective writing exercises contributed to their learning (Question 3). At the same time, however, slightly more than one third never consulted their reflections (Question 2) or did not use their reflections in preparing for class and or in-class tests (Question $4 \mathrm{~b}$ ). Moreover, a significant portion did not generalize the use of the strategy in other courses (Question 4a). 
ECSE 206

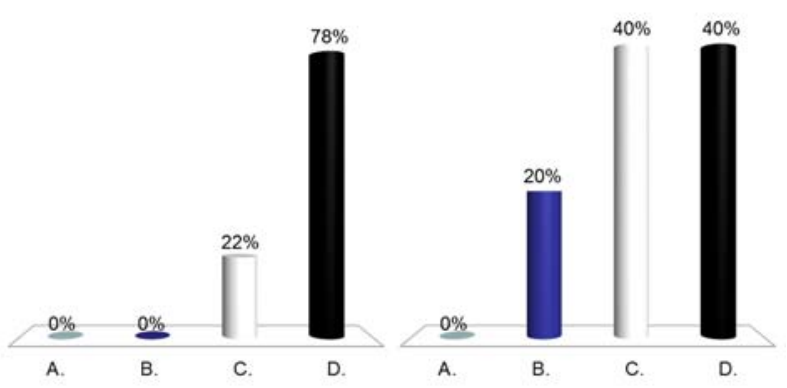

Question 1-I spend ... minutes doing the reflective writing exercises

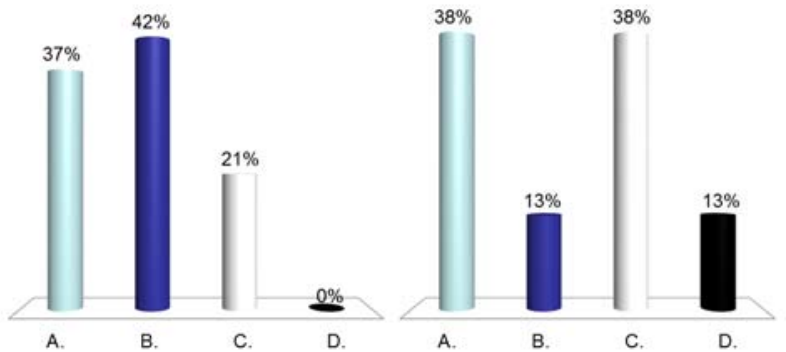

Question 2-I have referred to my reflective writings...

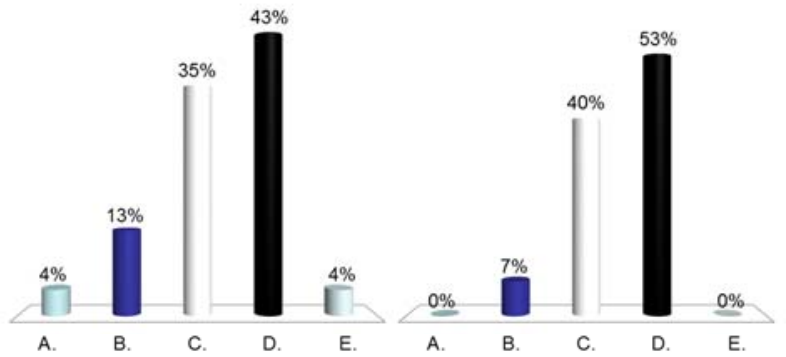

Question 3-Overall, the reflective writing exercises contribute to my learning

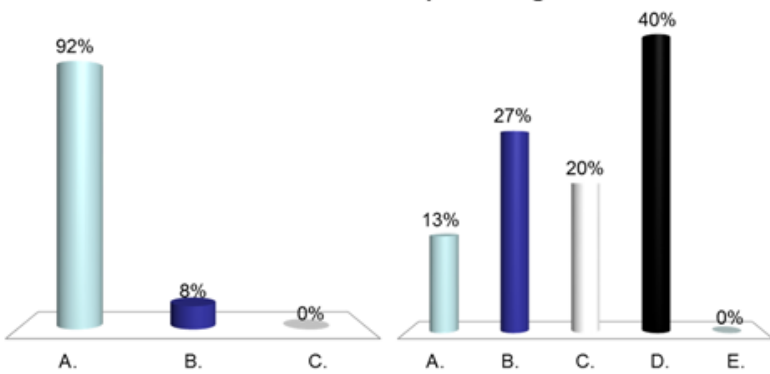

Question $4 a-I$ have tried reflective writing in other courses

Question $4 b-I$ used my reflective writing as a tool when preparing for class or assessments

Fig. 6. Results from student survey. Values given correspond to percentage of students.
The latter results suggest a correlation between the students' willingness to engage in the reflective writing exercises and the use of a flipped classroom approach: in most cases, ECSE 206 and ECSE 430/ECSE 540 were the only courses that students were taking that adopted the flipped classroom. This observation is consistent with studies that examine the impact of using a flipped classroom on student engagement and learning [12]. Based on the survey results, we also believe that the objectives and benefits of reflection need to be communicated better to the students, and reinforce the notion that opportunities for reflection should be further integrated in engineering curricula.

\section{CONCLUSIONS}

Based on our experience of implementing reflective writing exercises in the two courses, we observe a general correlation between a higher quality of reflection and better performance and/or improvement in grades on similar types of questions. Careful design and delivery of the reflective writing exercises are required in order to maximize gain. In particular, students should be guided in their reflection and it may be more beneficial to perform the SAs prior to assessments as the students can then take appropriate action, such as addressing gaps in knowledge and understanding. While reflection can be useful in all of the courses that a student is taking, we must ensure that they do not become overburdened with the process to the point that the quality of reflection degrades substantially. Further research is necessary to establish more concretely a causal relation between the quality of the reflection and student learning.

\section{Acknowledgements}

This investigation was supported in part by the eLATE Teaching and Learning Improvement Funds from the Faculty of Engineering at McGill University.

\section{References}

[1] Linda B. Nilson, Creating self-regulated learners: Strategies to strengthen students' self-awareness and learning skills. Sterling, VA: Stylus Publishing, 2013. \{ISBN: 978-1579228675\}

[2] Barry J. Zimmerman, "Theories of self-regulated learning and academic achievement: An overview and analysis," in Self-regulated learning and academic achievement, Barry J. Zimmerman and Dale H. Schunk (eds.) New York, NY: Routledge, 2013. \{eBook ISBN: 9781135659141\}

[3] Philip H. Winne and Allyson F. Hadwin, "Selfregulated learning and socio-cognitive theory," in International Encyclopedia of Education, $3^{\text {rd }}$ ed. Penelope Peterson, Eva Baker and Barry McGaw (eds.) Elsevier, 2010. \{ISBN: 978-0-08-044894-7\} 
[4] Susan A. Ambrose, "Undergraduate engineering curriculum: the ultimate design challenge,” Bridge, vol. 43, no. 2, pp. 16-23, 2013.

[5] Jennifer A. Turns, Brook Sattler, Ken Yasuhara, and Jim L. Borgford-Parnell, "Integrating reflection into engineering education," in Proc. $121^{\text {st }}$ ASEE Annual Conference \& Exposition (Indianapolis, IN; 15-18 June 2014), paper ID\#9230.

[6] Robin S. Adams, Jennifer Turns, and Cynthia J. Atman, "Educating effective engineering designers: the role of reflective practice,” Design Studies, vol. 24, no. 3, pp. 275-294, 2003.

[7] Jennifer A. Moon, Learning journals: a handbook for reflective practice and professional development. $\left(2^{\text {nd }}\right.$ Ed.) New York, NY: Routledge, 2006. \{ISBN 0-41540376\}

[8] Lawrence R. Chen and Maria Orjuela-Laverde, "Implementing reflective writing in large non-technical engineering courses," in Proc. CEEA Canadian Engineering Education Conf., CEEA18 (Vancouver, BC; 3-6 June 2018), paper ID\#050.
[9] David Benson and Haolin Zhu, "Student reflection, self-assessment and categorization of errors on exam questions as a tool to guide self-repair and profile student strengths and weaknesses in a course," in Proc. $122^{\text {nd }}$ ASEE Annual Conference \& Exposition (Seattle, WA; 14-17 June 2015), paper ID\#12366.

[10] Renee M. Clark and Samuel J. Dickerson, “Assessing the impact of reflective activities in digital and analog electronics courses," IEEE Transactions on Education, in press, 2019.

[11] David Kember, Jan McKay, Kit Sinclair, and Frances Kam Yuet Wong, "A four-category scheme for coding and assessing the level of reflection in written work," Assessment and Evaluation in Higher Education, vol. 33, no. 4, pp. 369-379, 2008.

[12] Alison S. Burke and Brian Fedorek, "Does “flipping” promote engagement?: a comparison of a traditional, online, and flipped class," Active Learning in Higher Education, vol. 18, no. 1, pp. 11-24, 2017. 